\title{
POTENTIAL EVIDENCE FOR THE ONSET OF ALFVÉNIC TURBULENCE IN TRANS-EQUATORIAL CORONAL LOOPS
}

\author{
I. De Moortel ${ }^{1}$, S. W. McIntosh ${ }^{2}$, J. Threlfall ${ }^{1}$, C. Bethge $^{3}$, And J. Liu ${ }^{4}$ \\ ${ }^{1}$ School of Mathematics and Statistics, University of St. Andrews, St. Andrews, Fife KY16 9SS, UK; ineke@mcs.st-and.ac.uk \\ ${ }^{2}$ High Altitude Observatory, National Center for Atmospheric Research, P.O. Box 3000, Boulder, CO 80307, USA; mscott@ucar.edu \\ ${ }^{3}$ Kiepenheuer Institute for Solar Physics, D-79104 Freiburg, Germany; bethge@kis.uni-freiburg.de \\ ${ }^{4}$ Earth and Space Science School, University of Science and Technology of China, NO. 96, JinZhai Road, Hefei, China \\ Received 2013 November 14; accepted 2013 December 28; published 2014 February 10
}

\begin{abstract}
This study investigates Coronal Multi-channel Polarimeter Doppler-shift observations of a large, off-limb, transequatorial loop system observed on 2012 April 10-11. Doppler-shift oscillations with a broad range of frequencies are found to propagate along the loop with a speed of about $500 \mathrm{~km} \mathrm{~s}^{-1}$. The power spectrum of perturbations travelling up from both loop footpoints is remarkably symmetric, probably due to the almost perfect north-south alignment of the loop system. Compared to the power spectrum at the footpoints of the loop, the Fourier power at the apex appears to be higher in the high-frequency part of the spectrum than expected from theoretical models. We suggest this excess high-frequency power could be tentative evidence for the onset of a cascade of the low-to-mid frequency waves into (Alfvénic) turbulence.
\end{abstract}

Key words: magnetohydrodynamics (MHD) - Sun: atmosphere - Sun: corona Sun: oscillations - turbulence - waves

Online-only material: color figures

\section{INTRODUCTION}

Modern day observations have revealed an abundance of waves, oscillations, and other quasi-periodic disturbances in many structures throughout the solar atmosphere (see, for example, the recent review by De Moortel \& Nakariakov 2012). Of particular interest to this Letter are the recent observations of propagating, transverse disturbances in structures such as prominences (Okamoto et al. 2007; Hershaw et al. 2011; Hillier et al. 2013), chromospheric spicules (De Pontieu et al. 2007; He et al. 2009a, 2009b; Jess et al. 2012), and mottles (Kuridze et al. 2012); coronal loops (McIntosh et al. 2011; Morton \& McLaughlin 2013); coronal rain (Antolin \& Verwichte 2011); and jets (Cirtain et al. 2007). Similar transverse perturbations were reported in Doppler-shift observations of large, off-limb, coronal loops by Tomczyk et al. (2007) and Tomczyk \& McIntosh (2009). Other observations show evidence for torsional waves in, e.g., chromospheric bright points (Jess et al. 2009), spicules (De Pontieu et al. 2012), and coronal holes (Banerjee et al. 2009) and the first evidence for sausage modes (Morton et al. 2011, 2012).

Many of these recently observed, footpoint-driven, oscillatory displacements are interpreted as "Alfvénic," although there has been substantial debate in the recent literature about the identification of some of these observations in terms of MHD wave theory (see, e.g., Erdélyi \& Fedun 2007; Van Doorsselaere et al. 2008 and the discussion in Section 3 of De Moortel \& Nakariakov 2012). What is of interest to this particular Letter, though, is that many of these observed waves and oscillations are reported to contain substantial amounts of energy, often comparable to the energy requirements of, at least, the quiet Sun corona (De Moortel \& Pascoe 2012; McIntosh \& De Pontieu 2012). Hence, a renewed interest in wave-heating mechanisms is gathering pace.

Although the observed waves and oscillations are suggested to contain a substantial amount of energy, one has to remember that this does not automatically imply a solution to the coronal heating problem; theoretical modeling now needs to address how these things lead to heating in the right locations at the right time (Parnell \& De Moortel 2012). For example, numerical simulations suggest that the damping of periodic Doppler-shift oscillations observed by Coronal Multi-channel Polarimeter (CoMP; Tomczyk et al. 2007; Tomczyk \& McIntosh 2009) can be explained due to the energy transfer from the footpointdriven observed transverse oscillation to an azimuthal Alfvén wave in the flux tube boundary (see, e.g., Pascoe et al. 2010, 2013 and references therein). Phase mixing of the Alfvén waves in the inhomogeneous tube boundaries could lead to enhanced dissipation and hence, local heating, but the viability of this has yet to be demonstrated. In the future, it is hoped that, for example, the extraordinary spatial and temporal resolution of ATST (now renamed the DKIST, Daniel K. Inouye Solar Telescope) will be sufficient to resolve both the mode coupling process in the narrow boundary layer of coronal loops and the subsequent phase mixing of the Alfvén waves.

In this Letter, we analyze CoMP observations of a large, diffuse, off-limb trans-equatorial loop and show that excess energy appears to be available in the high-frequency part of the power spectrum, which we interpret as tentative evidence of the onset of Alfvénic turbulence near the loop apex. The Letter is organized as follows: the setup is described in Section 2, the results are analyzed in Sections 3 and 4, and a discussion and conclusions are presented in Sections 5 and 6, respectively.

\section{OBSERVATIONS}

The present study focuses on large, off-limb coronal loops observed by CoMP on 2012 April 10 and 11. CoMP observes the off-limb solar corona from 1.05 to 1.25 solar radii. The intensity and Doppler velocities used in this Letter are based on the CoMP Fe XIII coronal $1074.7 \mathrm{~nm}$ emission line, with 4.5 arcsec pixel size and a $30 \mathrm{~s}$ cadence. Processing of the data 
(A) MLSO/CoMP Fe XIII 10747Å (Line Intensity) 04/10/2012

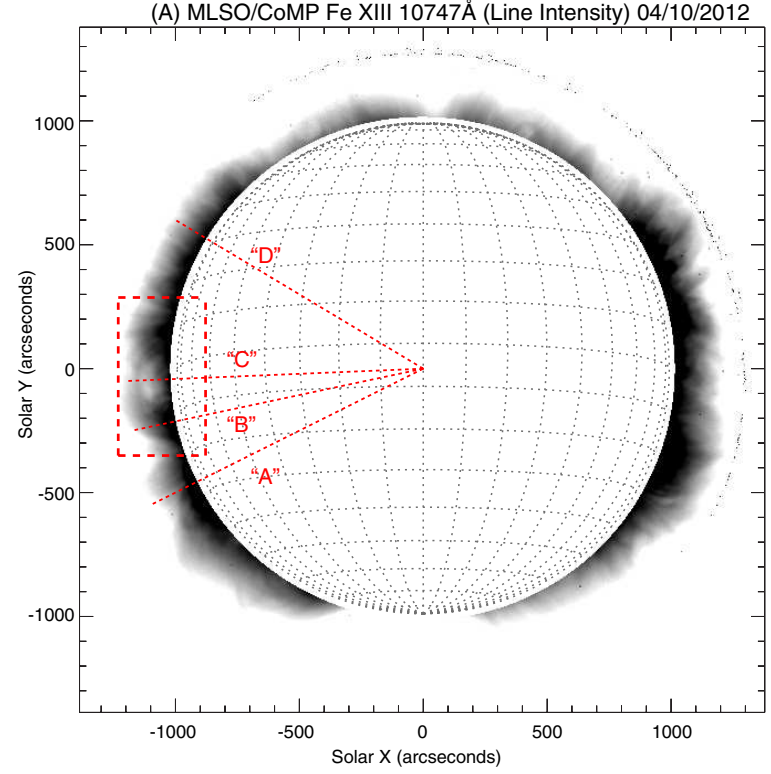

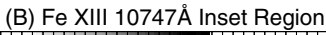

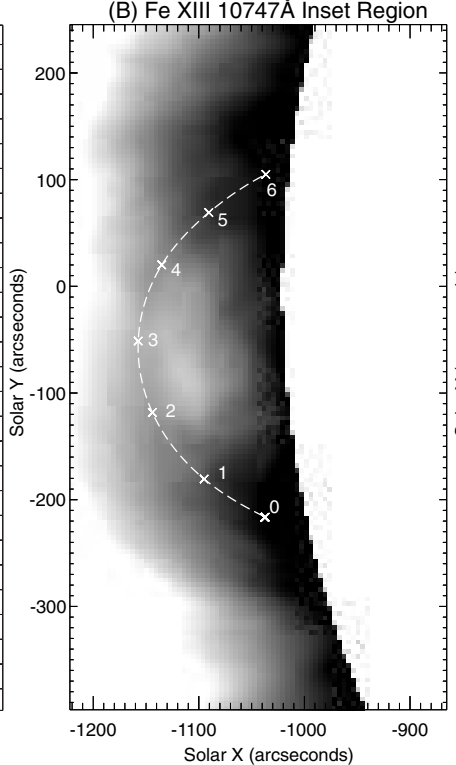

(C) STEREO EUVI-B 195A - 04/10/2012 17:35

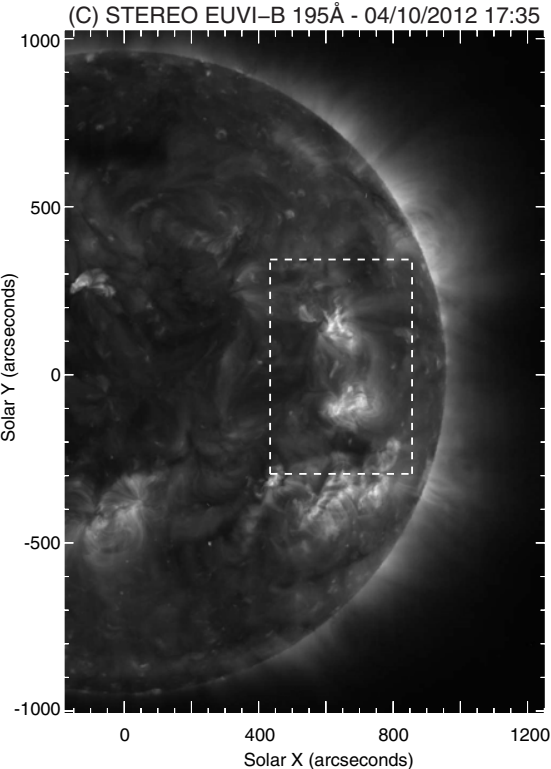

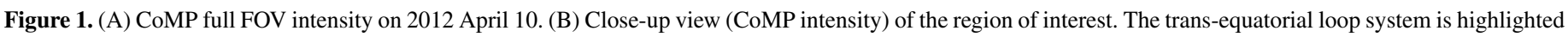
by the dashed arc. (C) Part of a STEREO/EUVI-B image taken on 2012 April 10.

(A color version of this figure is available in the online journal.)

involved correcting for an east-west trend in the velocities and co-aligning all frames using a cross-correlation technique. Note that the zero-point of the wavelength scale has been redefined by assuming that the mean Doppler shift over the field of view (FOV) is zero. Figure 1(A) shows the full CoMP FOV (intensity) on 2012 April 10 and the region of interest is outlined in the small box.

Figure 1(B) provides a close-up view of the region of interest. As in Threlfall et al. (2013), an arc is defined using six spline points and the coordinates of the arc are resampled to match the pixel size (4.46 arcsec for CoMP). The same coordinates are used for both the 2012 April 10 and 2012 April 11 datasets. The large, trans-equatorial loop system connects two small active regions. To provide context, Figure $1(\mathrm{C})$ is (part of) an image taken by STEREO/EUVI-B, which was observing the far side of the Sun (at a separation angle with Earth of about $118 \mathrm{deg}$ ) on 2012 April 10. The loop system connects the two active regions outlined by the box. This image shows how remarkably isolated and north-south aligned the system is, which will help reduce line-of-sight effects.

\section{TIME-DISTANCE ANALYSIS}

Figure 2(A) shows a time-distance plot along the arc highlighted in Figure 1. There is a clear herringbone pattern visible, indicating disturbances propagating up from both loop footpoints. The propagation speeds, estimated from the gradients of the diagonal bands (pink dashed lines), are of the order of $500 \mathrm{~km} \mathrm{~s}^{-1}$. These speeds are in agreement with those quoted by Tomczyk \& McIntosh (2009). They are substantially larger than the sound speed $\left(\sim 200 \mathrm{~km} \mathrm{~s}^{-1}\right.$ for $\left.T=1.5 \times 10^{6} \mathrm{~K}\right)$ and are of the order of the local Alfvén speed. For example, assuming a magnetic field strength of about $10 \mathrm{G}$ and a local number density of $10^{9} \mathrm{~cm}^{-3}$, would give an Alfvén speed of the order of $690 \mathrm{~km} \mathrm{~s}^{-1}$. The propagation speeds appear constant and very similar on both sides of the loop. Consistent with the earlier results from Tomczyk et al (2007) and Tomczyk \& McIntosh (2009), there is little evidence of downward propagation (as the ridges do not appear to carry on all the way from one footpoint to the other).

We can compute an order of magnitude estimate of the energy contained in the perturbations using

$$
F_{W}=\rho\left\langle v^{2}\right\rangle V_{\text {phase }},
$$

where $\rho$ is the density, $v$ the velocity amplitude, and $V_{\text {phase }}$ the phase speed of the waves. Again, using a typical number density of $10^{9} \mathrm{~cm}^{-3}, V_{\text {phase }}=500 \mathrm{~km} \mathrm{~s}^{-1}$ and a velocity amplitude of $0.65 \mathrm{~km} \mathrm{~s}^{-1}$ (root mean square amplitude of the velocity perturbations in our observations) gives an energy flux of $F_{W} \approx 350 \mathrm{erg} \mathrm{cm}^{-2} \mathrm{~s}^{-1}$. This estimate is of the same order as that obtained by Tomczyk \& McIntosh (2009) and several orders of magnitude too small to account for the heating of these loop structures. We refer the interested reader to Van Doorsselaere et al. (2008), Tomczyk \& McIntosh (2009) and Goossens et al. (2013) for a more detailed discussion on how this estimate depends on the interpretation of the observed velocity perturbations in terms of the various MHD waves but also to De Moortel \& Pascoe (2012) and McIntosh \& De Pontieu (2012) for a discussion on the effect of line-of-sight superposition on the energy budget.

Figure 2(B) shows the logarithm of the fast Fourier transform (FFT) power at each point along the loop. For reference, the vertical white lines correspond to periods of eight and three minutes. It is clear that, generally, most of the power is situated in low-frequency perturbations. Additionally, power at the loop apex appears higher, as we would expect from simple (linear) superposition of the perturbations traveling up from the footpoints and/or the effect of gravitational stratification (for which the velocity perturbations are expected to grow as $\rho^{-1 / 4}$, see e.g. Wright \& Garman 1998). For a temperature of 1.5 MK, the gravitational scale height is about $75 \mathrm{Mm}$ and our loop apex is situated about $125 \mathrm{Mm}$ above the solar limb so $e^{z_{\text {apex }} /(4 H)} \approx 1.5$. Hence, gravitational stratification could lead to an increase in amplitude at the apex by about $50 \%$. Linear superposition could potentially double the wave amplitudes so the two effects combined could lead to a total amplitude increase 

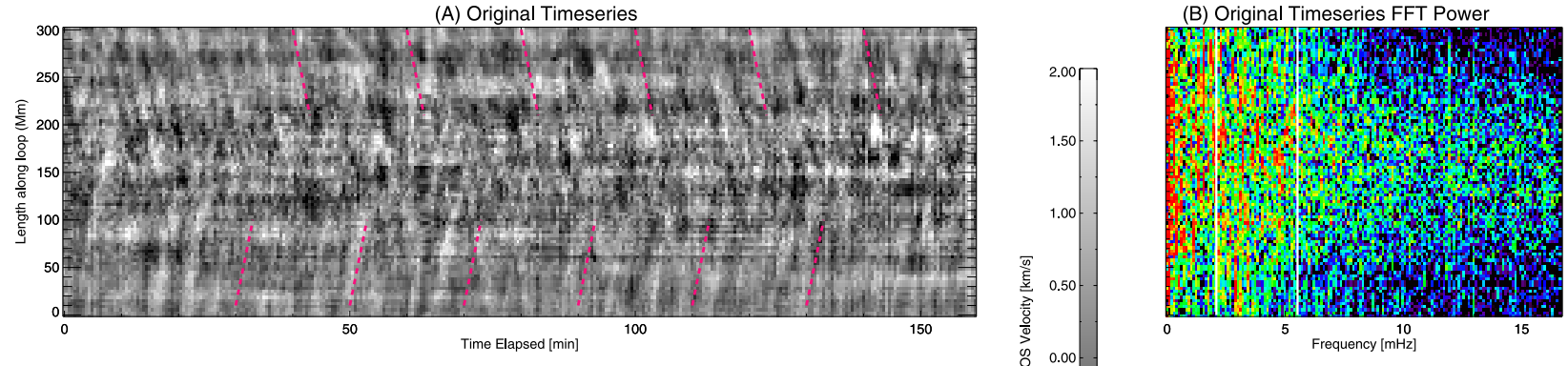

(C) Shuffled Timeseries

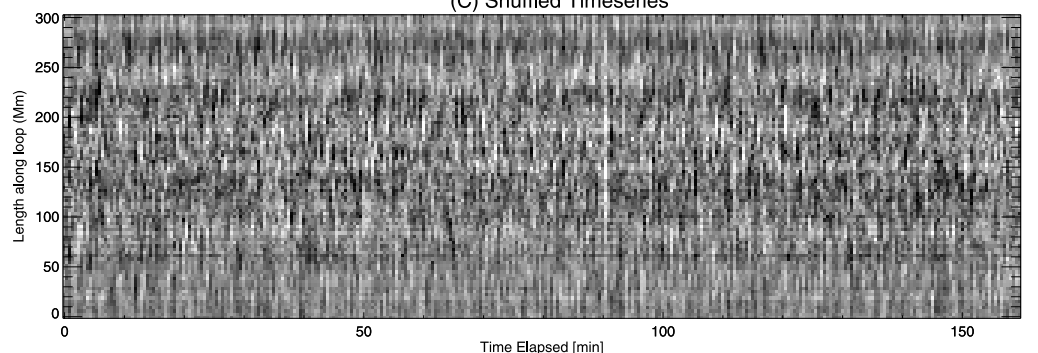

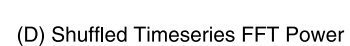

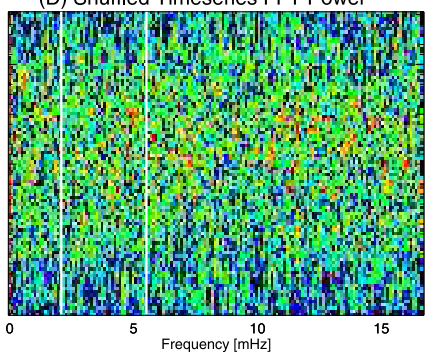

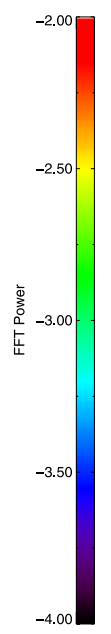

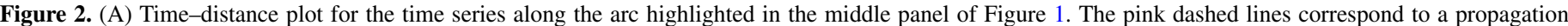

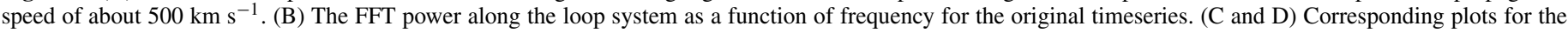
randomly shuffled time series.

(A color version of this figure is available in the online journal.)

by a factor of three at the loop apex although this is more than likely a (strong) upper bound.

Looking at the distribution of Fourier power more carefully reveals an intriguing pattern; at the footpoints, power appears to be concentrated in the low-frequency part of the domain, peaking around five minutes, as previously found by Tomczyk et al. (2007) and Tomczyk \& McIntosh (2009). Almost no power is present in the higher-frequency part of the domain at the loop footpoints. The distribution of power at both footpoints is also remarkably similar. At the apex, on the other hand, there is still a lot of power in the low-frequency range, but there now appears to be significant power in the high-frequency range.

As a quick, zeroth-order null test, we randomly reshuffle the observed time series at every point along the loop and the corresponding time-distance plot is shown in Figure 2(C). There is no longer any evidence of the criss-cross, herringbone pattern associated with coherently counter-propagating perturbations. In the corresponding Fourier power plot (Figure 2(D)), the intriguing pattern observed in the Fourier power has equally disappeared and power is more or less uniformly distributed over the entire frequency range at each point along the loop, as we would expect for a randomly shuffled timeseries.

Although not shown here, the 2012 April 11 data result in an almost identical time-distance figure and the same pattern in the FFT power plot. Given the isolation of the loop system (as evident from the STEREO/EUVI-B image in Figure 1) we can be relatively sure that we are studying the same loop system on 2012 April 10 and 11 and hence, both the presence (i.e., generation and propagation) of these waves and the excess in $\mathrm{HF}$ power appear very robust features.

\section{HIGH FREQUENCY POWER AT THE LOOP APEX}

From Figure 2, there appears to be more high-frequency power at the loop than one would expect given the power present at the loop footpoints. To investigate this in more detail, we integrate the FFT power over three different parts of the frequency domain. This integrated power as a function of loop length is plotted in Figures 3(A) and (B) for the original and shuffled time series, respectively. Low-frequency (LF; black) power is taken to be at periods longer than eight minutes, medium-frequency (MF; blue) power corresponds to three to eight minutes and high-frequency (HF; red) power to periods less than three minutes. To allow easy comparison, each of the graphs has been normalized to its own mean. Note that the three parts of the frequency spectrum of the reshuffled timeseries have roughly the same mean, as would be expected from the random shuffling.

All three curves (for the original timeseries) show higher power at the loop apex, as already noted earlier. However, comparing the curves in detail shows that the behavior of the Fourier power at high frequencies (red curve) does indeed appear different from the LF-MF part of the spectrum. The HF power appears to increase along the loop at a greater rate than the LF and MF power and, at the apex, clearly peaks above the LF and MF curves. This excess in HF power at the loop apex is especially clear when comparing with the corresponding curves for the randomly shuffled time series. These (shuffled) curves show roughly the same behavior along the loop for the three different parts of the frequency domain, as expected from the random nature of the timeseries shuffling. Again we note that the behavior in both loop legs is remarkably symmetric, for all parts of the frequency domain.

To confirm our findings, we now look at the Fourier power as a function of frequency, plotted in Figures 3(C) and (D). The black dashed lines correspond to the lower parts of the loop (with the power integrated over the first and last 20 grid points along the loop, respectively). The solid black line represents the average of the footpoint power (i.e., of the two dashed curves) whereas the solid red line represents the FFT power at the apex of the loop (integrated over the middle 20 grid points). To be able to compare the different curves directly, the Fourier power at each position along the loop was normalized to the total FFT power at that position before integrating over the relevant grid points. This normalization removes the increase in FFT power near the loop apex due to superposition and/or gravitational stratification but does not alter the gradient of the power as a 
(A) Original Timeseries

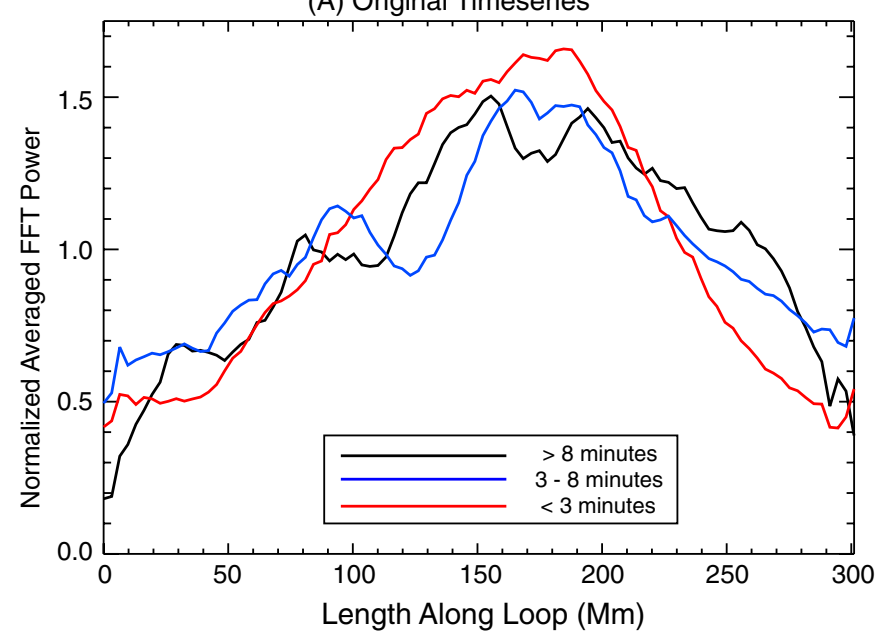

(C) Original Timeseries

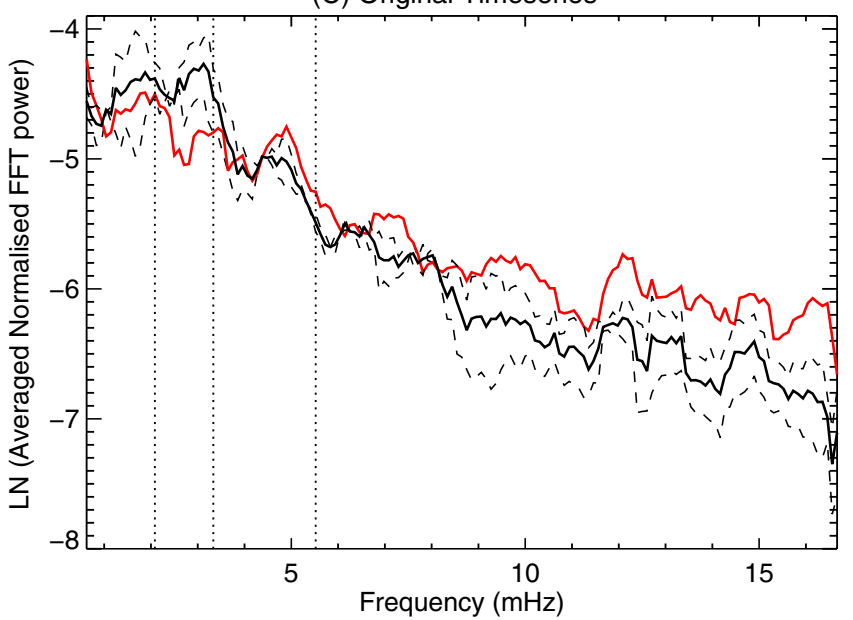

(B) Shuffled Timeseries

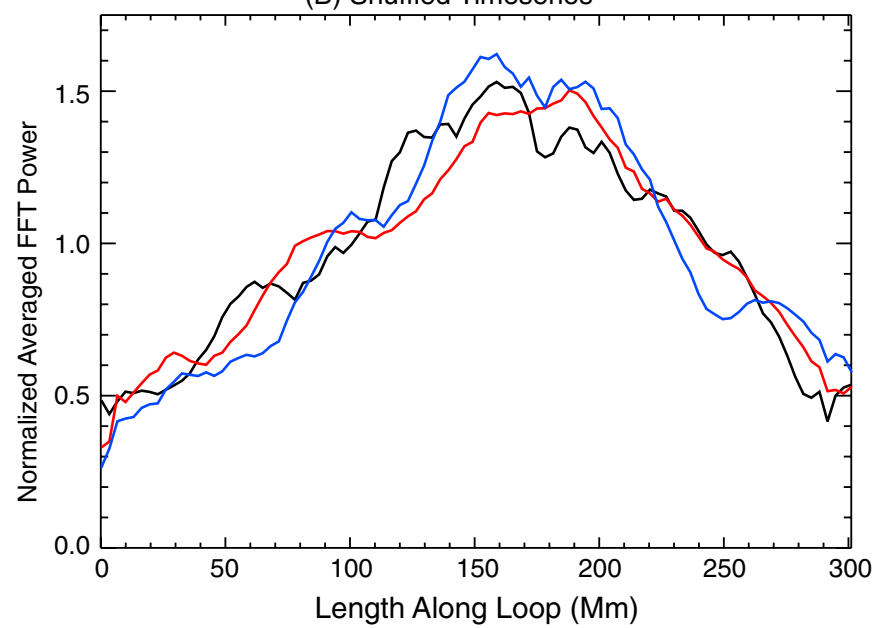

(D) Shuffled Timeseries

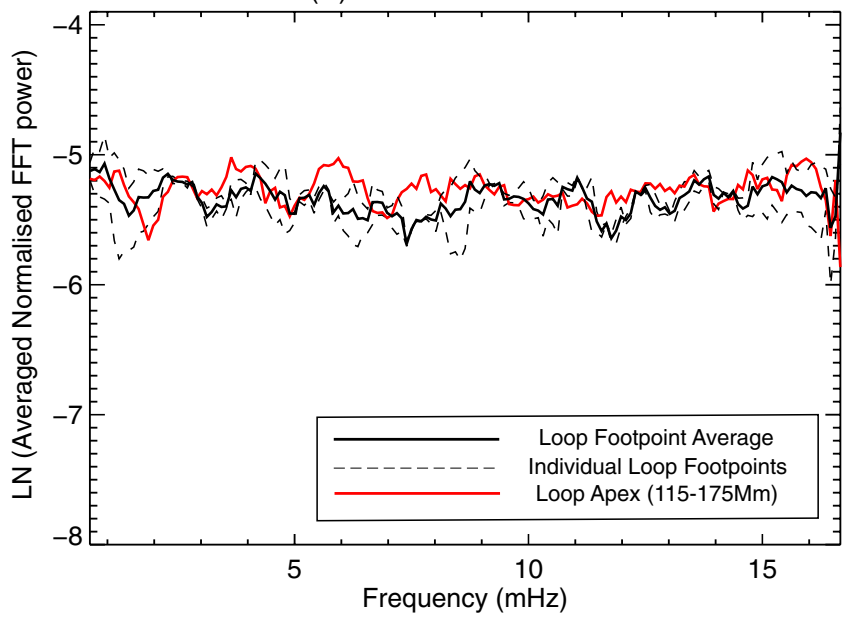

Figure 3. (A) Integrated (smoothed) FFT power for low (black; longer than eight minutes), medium (blue; three to eight minutes), and high (red; shorter than three minutes) frequencies for the 2012 April 10 time series. Each of the curves has been normalized to its own mean. (C) The natural logarithm of the averaged, normalized (smoothed) FFT power at both footpoints (dashed black lines) and the apex (solid red line) for the original time series. The solid black line corresponds to the average of the FFT power at the footpoints. For reference, the vertical lines are at periods of three, five, and eight minutes. (B and D) Corresponding results for the randomly shuffled time series.

(A color version of this figure is available in the online journal.)

function of frequency, which is the property in which we are interested. The vertical lines indicate periods of three, five, and eight minutes.

The remarkable symmetry of the loop is again obvious from the near-identical FFT power at both loop footpoints. Also, for each part of the loop, power is lower at high frequencies, as was already evident from Figure 2.

Looking at the gradient of the FFT power at the apex (as a function of frequency), it is clearly different from the footpoints, with different damping rates in the LF/MF and HF regimes: the normalized FFT power at the apex in the LF-MF range is less than at the footpoints but is higher than the average footpoint power in the HF range. As a comparison, exactly the same procedure was followed for the randomly shuffled time series (Figure 3(D)). As expected, the power is evenly distributed across frequencies in the shuffled case.

\section{DISCUSSION}

In the previous section, we compared the results for the original time series (2012 April 10) with the corresponding, randomly shuffled time series as a basic, zeroth order null test to establish the reliability of the excess HF power near the loop apex. The same test was done for the 2012 April 11 dataset, with the same results. As a further indication that this is indeed a real result, we repeat the analysis on CoMP observations taken at the same time but at different locations around the limb. We use simple radial cuts, shown by the dotted lines in Figure 1(A) and labeled "A"-"D." Here, cuts A and D are chosen at random to go through a section of the off-limb corona with a brightness comparable to that of the apex of the loop system we are studying. Cut B goes through the lower loop leg whereas cut $\mathrm{C}$ intersects with the apex of the loop. From Figure 4 it is clear that only cut $\mathrm{C}$, which goes through the apex of the loop system, shows the same characteristic pattern with high FFT power in the $\mathrm{HF}$ range of the spectrum. For all the other cuts (A, B, and D), the HF power is very low. This comparison gives us confidence that the high FFT power in the HF part of the spectrum is a real property of the trans-equatorial loop system rather than being caused by low signal-to-noise or other instrumental effects.

Since the first observation of these propagating Doppler velocity perturbations by Tomczyk et al. (2007), it has been clear that these oscillations must undergo significant damping as the perturbations are rarely seen to reach to the opposite loop 


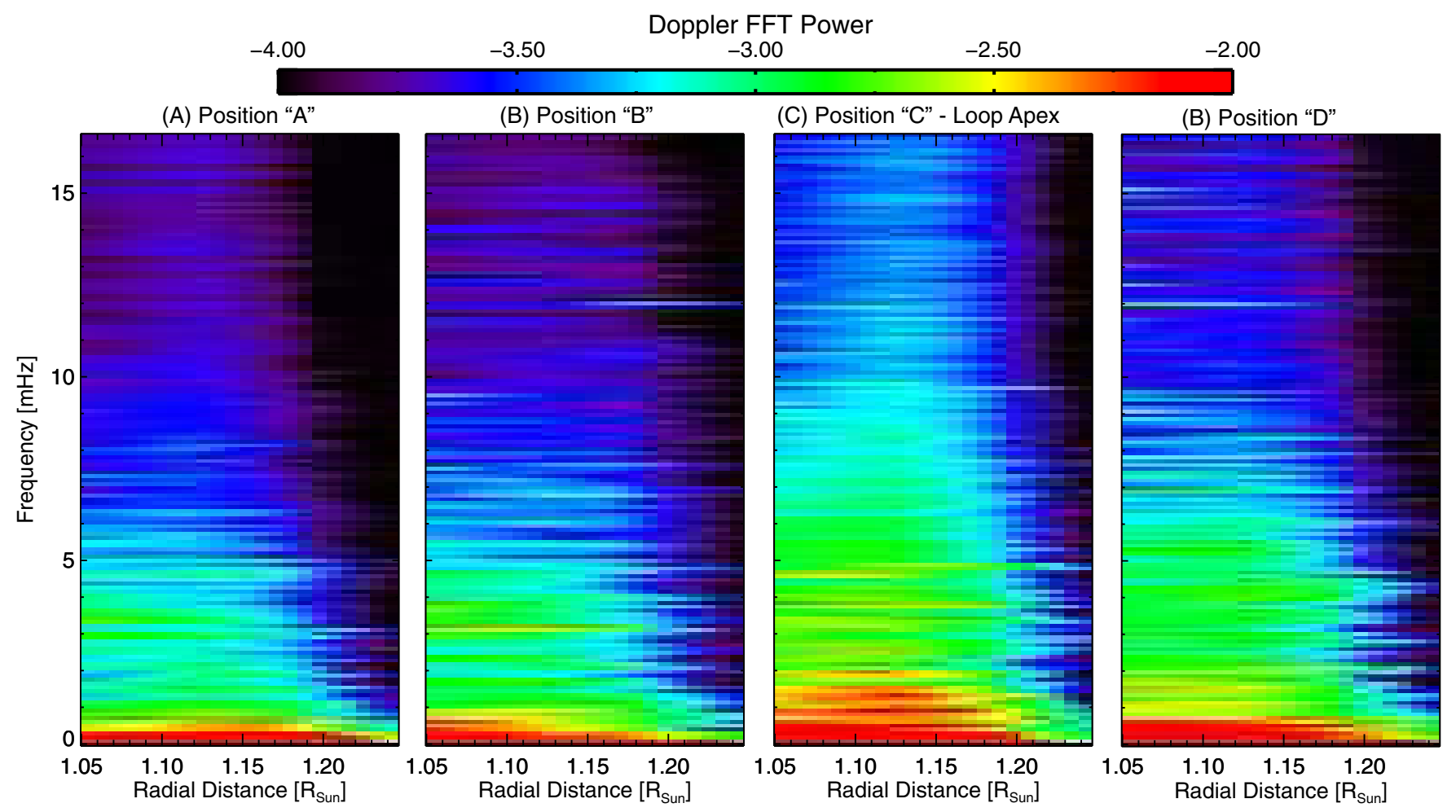

Figure 4. FFT power as a function of radial distance for the four radial cuts outlined by the dotted lines and labeled "A" to "D" in Figure 1(A).

(A color version of this figure is available in the online journal.)

footpoint (Tomczyk \& McIntosh 2009). Numerical simulations have suggested that the damping of the periodic Doppler shift oscillations can be explained in terms of mode coupling: energy is transferred from the footpoint-driven (observed) transverse oscillations to an azimuthal Alfvén wave in the flux tube boundary. This mode coupling process has been investigated in great detail recently (see, e.g., Pascoe et al. 2010; Terradas et al. 2010; Verth et al. 2010; Pascoe et al. 2013 and references therein).

Of interest to this current study is that mode coupling has a distinct frequency filtering effect, as it is more efficient for higher frequencies (Pascoe et al. 2010; Terradas et al. 2010; Verth et al. 2010). Hence, one expects the higher frequencies to damp fastest, exactly the opposite of what we found in the oscillations analyzed in this Letter. As a rough guide, we estimate the amount of damping by comparing the FFT power at the loop apex with the maximum power at the footpoints. The LF/MF FFT power at the apex is roughly 1.1-1.5 times higher than at the footpoints. If we assume the wave amplitudes could have grown by up to a factor of three by the time they reach the apex (due to gravitational stratification and/or superposition), the waves might have damped by as much as $60 \%-65 \%$ in the LF/MF regime. The HF FFT power at the apex, on the other hand, is about three times the FFT power at the footpoints and hence, the damping would only be about $40 \%$.

One would expect higher frequency waves to damp at least as quickly as their LF counterparts, if not quicker, as in, for example, the mode coupling mechanism. A recent report by Morton et al. (2013) on observations of transverse perturbations in the chromosphere did indeed find that higher frequency waves appeared to damp quicker. This leaves us with the possibility that some of the HF power is actually generated as the waves travel along the loops. We suggest that the excess power in the HF spectrum at the apex of the loop could be tentative evidence for the onset of (Alfvénic) turbulence. Such a scenario of wave turbulence was recently modeled by Van Ballegooijen et al. (2011) and Asgari-Targhi \& Van Ballegooijen (2012) and has been studied extensively in a solar wind context (e.g., Parker 1991; Cranmer \& van Ballegooijen 2005; Suzuki \& Inutsuka 2005; Cranmer et al. 2007; Verdini et al. 2010; Perez \& Chandran 2013). One possible scenario (as suggested by McIntosh et al. 2013) is that slower propagating compressive (intensity) perturbations interact with the faster, transverse (Doppler shift) perturbations, possibly leading to reflection and counter-propagating waves, driving Alfvénic turbulence. Indeed, Threlfall et al. (2013) recently demonstrated that such slow intensity perturbations co-exist with faster, transverse Doppler-shift perturbations. The next generation of high spatial and temporal resolution solar observations such as, for example, DKIST or the Coronal Solar Magnetism Observatory, will hopefully be able to resolve such cascades to smaller scales and hence shed light on the scenario suggested here.

\section{CONCLUSIONS}

In this Letter, we analyzed CoMP Doppler-shift observations of a large, trans-equatorial loop system. The loops connect two small active regions which are almost directly north-south aligned. We found a remarkably symmetric, broadband spectrum of Doppler-shift oscillations propagating up from both footpoints at speeds of about $500 \mathrm{~km} \mathrm{~s}^{-1}$. Looking at the distribution of the FFT power of the oscillations along the loop, more high-frequency power than expected is present at the loop apex (compared to, e.g., mechanisms such as mode coupling). We tentatively suggest this excess high-frequency power could be evidence for the onset of (Alfvénic) turbulence as the 
waves propagate along the loop. In a forthcoming paper, we use high-resolution numerical simulations to model the proposed scenario of turbulence driven by a combination of rapidly propagating Alfvénic (transverse) velocity perturbations and slow, longitudinal (density) perturbations (I. De Moortel et al. 2014, in preparation).

The authors would like to thank Bart De Pontieu for helpful discussions. I.D.M. acknowledges support from a Royal Society University Research Fellowship. The research leading to these results has also received funding from the European Commission Seventh Framework Programme (FP7/20072013) under the grant agreements SOLSPANET (project No. 269299, www.solspanet.eu/solspanet). NCAR is sponsored by the National Science Foundation. CoMP data can be found at http://mlso.hao.ucar.edu/. S.M.C. acknowledges support from NASA contracts NNX08BA99G, NNX11AN98G, NNM12AB40P, and NNG09FA40C (IRIS).

\section{REFERENCES}

Antolin, P., \& Verwichte, E. 2011, ApJ, 736, 121

Asgari-Targhi, M., \& Van Ballegooijen, A. A. 2012, ApJ, 746, 81

Banerjee, D., Pérez-Suárez, D., \& Doyle, J. G. 2009, A\&A, 501, L15

Cirtain, J. W., Golub, L., Lundquist, L., et al. 2007, Sci, 318, 1580

Cranmer, S. R., \& van Ballegooijen, A. A. 2005, ApJS, 156, 265

Cranmer, S. R., van Ballegooijen, A. A., \& Edgar, R. J. 2007, ApJS, 171, 520

De Moortel, I., \& Nakariakov, V. M. 2012, Phil. Trans. R. Soc. A, 370, 3193

De Moortel, I., \& Pascoe, D. J. 2012, ApJ, 746, 31

De Pontieu, B., Carlsson, M., Rouppe van der Voort, L. H. M., et al. 2012, ApJL, 752, L12

De Pontieu, B., McIntosh, S. W., Carlsson, M., et al. 2007, Sci, 318, 1574

Erdélyi, R., \& Fedun, V. 2007, Sci, 318, 1572
Goossens, M., Van Doorsselaere, T., Soler, R., \& Verth, G. 2013, ApJ, 768, 191 He, J.-S., Marsch, E., Tu, C.-Y., \& Tian, H. 2009a, ApJL, 705, L217

He, J.-S., Tu, C.-Y., Marsch, E., et al. 2009b, A\&A, 497, 525

Hershaw, J., Foullon, C., Nakariakov, V. M., \& Verwichte, E. 2011, A\&A, 531, A53

Hillier, A., Morton, R. J., \& Erdélyi, R. 2013, ApJL, 779, L16

Jess, D. B., Mathioudakis, M., Erdélyi, R., et al. 2009, Sci, 323, 1582

Jess, D. B., Pascoe, D. J., Christian, D. J., et al. 2012, ApJL, 744, L5

Kuridze, D., Morton, R. J., Erdélyi, R., et al. 2012, ApJ, 750, 51

McIntosh, S. W., Bethge, C., Threlfall, J., et al. 2013, arXiv:1311.2538

McIntosh, S. W., \& De Pontieu, B. 2012, ApJ, 761, 138

McIntosh, S. W., De Pontieu, B., Carlsson, M., et al. 2011, Nat, 475, 477

Morton, R. J., Erdélyi, R., Jess, D. B., \& Mathioudakis, M. 2011, ApJL, 729, L18

Morton, R. J., \& McLaughlin, J. A. 2013, A\&A, 553, L10

Morton, R. J., Verth, G., Hillier, A., \& Erdélyi, R. 2013, arXiv:1310.4650

Morton, R. J., Verth, G., Jess, D. B., et al. 2012, NatCo, 3, 1315

Okamoto, T. J., Tsuneta, S., Berger, T. E., et al. 2007, Sci, 318, 1577

Parker, E. N. 1991, ApJ, 372, 719

Parnell, C. E., \& De Moortel, I. 2012, Phil. Trans. R. Soc. A, 370, 3217

Pascoe, D. J., Hood, A. W., De Moortel, I., \& Wright, A. N. 2013, A\&A, 551, A40

Pascoe, D. J., Wright, A. N., \& De Moortel, I. 2010, ApJ, 711, 990

Perez, J. C., \& Chandran, B. D. G. 2013, ApJ, 776, 124

Suzuki, T. K., \& Inutsuka, S.-I. 2005, ApJL, 632, L49

Terradas, J., Goossens, M., \& Verth, G. 2010, A\&A, 524, A23

Threlfall, J., De Moortel, I., McIntosh, S. W., \& Bethge, C. 2013, A\&A, 556, A124

Tomczyk, S., \& McIntosh, S. W. 2009, ApJ, 697, 1384

Tomczyk, S., McIntosh, S. W., Keil, S. L., et al. 2007, Sci, 317, 1192

Van Ballegooijen, A. A., Asgari-Targhi, M., Cranmer, S. R., \& DeLuca, E. E. 2011, ApJ, 736, 3

Van Doorsselaere, T., Nakariakov, V. M., \& Verwichte, E. 2008, ApJL, 676, L73

Verdini, A., Velli, M., Matthaeus, W. H., Oughton, S., \& Dmitruk, P. 2010, ApJL, 708, L116

Verth, G., Terradas, J., \& Goossens, M. 2010, ApJL, 718, L102

Wright, A. N., \& Garman, A. R. 1998, JGR, 103, 2377 